\title{
The effects of oscillating boundary conditions on thermal ignition
}

\author{
Matthew Berry ${ }^{1} \quad$ Mark Nelson ${ }^{2} \quad$ Brian Monaghan ${ }^{3}$ \\ Ben Whale ${ }^{4}$
}

(Received 20 January 2020; revised 12 May 2020)

\begin{abstract}
We investigate the effect that oscillating ambient temperatures have on the ignition times of supercritical stockpiles. Large stockpiles are exposed to seasonal and diurnal temperature variation. We analyse the effects of seasonal temperature variation. When considering ignition within a year of construction, stockpiles built in spring ignited with a lower critical parameter than those built at other times. Consequently, seasonal temperature variation needs to be accounted for when predicting stockpile ignition times.
\end{abstract}

DOI:10.21914/anziamj.v61i0.15035, C Austral. Mathematical Soc. 2020. Published 2020-06-16, as part of the Proceedings of the 14th Biennial Engineering Mathematics and Applications Conference. ISSN 1445-8810. (Print two pages per sheet of paper.) Copies of this article must not be made otherwise available on the internet; instead link directly to the DOI for this article. 


\section{Contents}

1 Introduction

C46

2 Mathematical model

C47

3 Results

C49

4 Numerical validation

C54

5 Conclusion

C56

\section{Introduction}

The Basic Oxygen Steelmaking process produces excess dust. This dust is collected and processed into a filter cake, which is used to construct large stockpiles. In these stockpiles, temperatures of approximately $1000^{\circ} \mathrm{C}$ have been observed. However, not all stockpiles reach this temperature, even when built in an analogous manner. We seek to understand the causal links as to why some of the stockpiles fail to reach these temperatures. High temperatures lead to sintering of the filter cake, where the underlying structure changes. The new structure increases the strength and particle size, which allows it to be recycled on site [2]. High temperatures are desirable to improve the recycling process.

The stockpiles are left exposed to seasonal and diurnal ambient temperature variations. We investigate the effects that seasonal variations have on the propensity of these stockpiles to oxidise. The oxidation reactions are exothermic and the behaviour is similar to stockpiles seen in the spontaneous combustion literature [1]. Under this framework we define ignition as when the stockpiles reach a certain temperature.

Although the theory of spontaneous combustion is well established, there has been comparatively little work investigating the effects of periodic boundary 
Table 1: Parameter values.

\begin{tabular}{llrr} 
Parameter & Symbol & Value & Units \\
\hline Activation Energy & $\mathrm{E}$ & $8.91 \times 10^{4}$ & $\mathrm{~J} \mathrm{~mol}^{-1}$ \\
Ideal Gas Constant & $\mathrm{R}$ & 8.314 & $\mathrm{~J} \mathrm{~mol}^{-1} \mathrm{~K}^{-1}$ \\
Mean Ambient Temperature & $\mathrm{T}_{\mathrm{a}}$ & 290 & $\mathrm{~K}$ \\
Temperature oscillation amplitude & $\mathrm{T}_{\mathrm{o}}$ & 5 & $\mathrm{~K}$ \\
Height & $\mathrm{L}_{y}$ & 10 & $\mathrm{~m}$ \\
Length & $\mathrm{L}_{x}$ & 20 & $\mathrm{~m}$ \\
Thermal Diffusivity & $\alpha$ & 30 & $\mathrm{~m}^{2} \mathrm{year}^{-1}$ \\
Oscillation Frequency & $\omega_{Y}$ & 1 & $\mathrm{year}$ \\
Phase Shift & $\phi$ & 0 & - \\
Heat of Reaction & $\mathrm{Q}$ & - & $\mathrm{J} \mathrm{kg}^{-1}$ \\
Density & $\rho$ & - & $\mathrm{kg} \mathrm{m}^{-3}$ \\
Specific heat & $\mathrm{c}$ & - & $\mathrm{J} \mathrm{kg}^{-1} \mathrm{~K}^{-1}$ \\
Thermal Conductivity & $\mathrm{k}$ & - & $\mathrm{J} \mathrm{m}^{-1} \mathrm{year}^{-1}$ \\
Pre-exponential factor & $\mathrm{A}$ & - & $\mathrm{year}^{-1}$
\end{tabular}

oscillations. We consider the model used by Novozhilov [3]. This contains a single Arrhenius reaction and a sinusoidal boundary condition. We extend this model to a two-dimensional domain, which will provide a stepping stone to a three-dimensional model. Roy [4] used a similar model in a two dimensional domain. However, this only included a sinusoidal boundary condition on one boundary; this is indicative of modelling an experimental procedure rather than modelling exposed stockpiles.

\section{Mathematical model}

The temperature $\mathrm{T}$ measured in Kelvin $(\mathrm{K})$, is modelled using a reactiondiffusion equation with an Arrhenius reaction term

$$
\rho c \frac{\partial T}{\partial t}=k\left(\frac{\partial^{2} T}{\partial x^{2}}+\frac{\partial^{2} T}{\partial y^{2}}\right)+Q \rho A \exp \left(\frac{-E}{R T}\right),
$$


where the parameters are defined in Table 1 . We consider a rectangular domain with $0 \leqslant x \leqslant L_{x}$ and $0 \leqslant y \leqslant L_{y}$. A no flux boundary condition is considered on the base $y=0$, and the boundary condition on the other sides is

$$
\mathrm{T}=\mathrm{T}_{\mathrm{a}}-\mathrm{T}_{\mathrm{o}} \sin \left(\frac{2 \pi \mathrm{t}}{\omega_{\mathrm{Y}}}+\phi\right) .
$$

The phase shift $\phi$ corresponds to when the stockpile is constructed during the year. We consider two cases: $T_{0}=0$, where there are no oscillations, which we refer to as a static boundary condition; and $\mathrm{T}_{\mathrm{o}} \neq 0$, where there are temperature oscillations, which we refer to as a dynamic boundary condition. To obtain dimensionless variables these equations are scaled by

$$
\begin{aligned}
\mathrm{y}^{*} & =\frac{\mathrm{y}}{\mathrm{L}_{\mathrm{y}}}, & \mathrm{x}^{*} & =\frac{\mathrm{x}}{\mathrm{L}_{\mathrm{y}}}, \\
\mathrm{t}^{*} & =\frac{\alpha}{\mathrm{L}_{\mathrm{y}}^{2}} \mathrm{t}, & \mathrm{u} & =\frac{\mathrm{E}}{\mathrm{RT}_{\mathrm{a}}^{2}}\left(\mathrm{~T}-\mathrm{T}_{\mathrm{a}}\right),
\end{aligned}
$$

where $\alpha=k /(c \rho)$. For convenience we only consider the scaled equations and drop the $*$ superscript. Equation (1) reduces to

$$
\frac{\partial u}{\partial t}=\frac{\partial^{2} u}{\partial x^{2}}+\frac{\partial^{2} u}{\partial y^{2}}+\delta \exp \left(\frac{u}{1+\varepsilon u}\right),
$$

where $\varepsilon=T_{a} R / E$ and

$$
\delta=\mathrm{L}_{\mathrm{y}}^{2} \frac{\mathrm{Q} \rho \mathrm{A}}{\mathrm{k}} \exp \left(\frac{-\mathrm{E}}{\mathrm{RT}_{\mathrm{a}}}\right) \frac{\mathrm{E}}{\mathrm{RT}_{\mathrm{a}}^{2}}
$$

is the Frank-Kamanetskii (FK) parameter. The boundary conditions become

$$
\left.\frac{\partial u}{\partial y}\right|_{y=0}=0 \text { and } u=u_{o} \sin \left(\frac{2 \pi t}{\omega}+\phi\right) \text {, }
$$

where $u_{o}=\left(E / R T_{a}\right)\left(T_{o} / T_{a}\right)$ and $\omega=\alpha \omega_{Y} / L_{y}^{2}$. 
The parameter $\varepsilon$ is often small, $\varepsilon \ll 1$. This allows us to approximate $1+\varepsilon \mathfrak{u} \approx 1$ for small enough values of $u$. When $u$ becomes large we have ignition occurring. To simplify the notation we state the approximation as $\varepsilon=0$. This allows for an analytic form of the steady state solution.

For the purpose of our analysis we need to define an ignition criteria. We define the ignition time $t_{\mathrm{ig}}$ as the time taken for the maximum temperature within the stockpile to reach $u=100$. This scaled temperature corresponds to a temperature of approximately $800^{\circ} \mathrm{C}$. In the practical application this temperature is considered sufficient for ignition. We consider a stockpile to be subcritical if the scaled temperature never attains a maximum of $u=100$.

With the absence of an analytic solution, the critical parameters are determined numerically. To obtain exact values, the numerical model needs to be run for an infinite amount of time. Since this is not possible, a reasonable cut-off time needs to be selected. For the one-dimensional model cut-off times of $t_{f}=0.3,1,10,100$ were selected. In the two dimensional case we used the same cut-off times as the one-dimensional case, except we excluded $t_{f}=100$ due to high computational time. With the prescribed parameters, $t_{f}=0.3$ corresponds to one year. This is a realistic cut-off time for the types of stockpiles under investigation. The critical parameter $\delta_{\text {cr }}$ is defined such that if $\delta>\delta_{\mathrm{cr}}$, then ignition is achieved before the cut-off time.

\section{Results}

We initially consider two specific cases, $\mathrm{L}_{x}=2 \mathrm{~L}_{y}$ and $\mathrm{L}_{x}=\infty$. For the case where $\mathrm{L}_{x}=2 \mathrm{~L}_{y}$ the reflective condition prescribed at the boundary $y=0$ means that we can instead solve the problem on the square with non-dimensional length $\mathrm{L}_{x}=2$. This solution restricted to the domain $(0,2) \times(0,1)$ will solve our equation. For the approximation $\varepsilon=0$ and Dirichlet boundary conditions, the critical FK value is $\delta_{\mathrm{cr}}=1.7$ [1]. For $\mathrm{L}_{x} \gg \mathrm{L}_{y}$ we approximate the model by a one-dimensional model without diffusion in the $x$ direction. For the approximation $\varepsilon=0$ and the static 
Table 2: The critical FK parameter $\delta_{\text {cr }}$ in a one dimensional stockpile for different cut-off times, with $u_{0}=0.637, \omega=0.3$ and $\phi=0$ for the cases with oscillations.

\begin{tabular}{lccccc}
$\varepsilon$ & Oscillations & $\mathrm{t}_{\mathrm{f}}=0.3$ & $\mathrm{t}_{\mathrm{f}}=1$ & $\mathrm{t}_{\mathrm{f}}=10$ & $\mathrm{t}_{\mathrm{f}}=100$ \\
\hline 0 & no & 3.701 & 1.582 & 0.899 & 0.877 \\
0 & yes & 3.428 & 1.537 & 0.895 & 0.875 \\
0.027 & no & 3.910 & 1.658 & 0.927 & 0.904 \\
0.027 & yes & 3.639 & 1.612 & 0.924 & 0.901
\end{tabular}

boundary condition, the critical FK value is $\delta_{\mathrm{cr}}=0.88$ [1]. Critical FK values obtained from our simulations, displayed in Table 2, agree with values stated by Bowes [1] to the given number of significant figures.

Table 2 suggests that for the larger cut-off times $\left(t_{f}=10,100\right)$ the dynamic boundary condition does not have a significant influence on the critical FK parameter, with a slight reduction observed when the oscillations are added. However, there is a significant difference in the ignition times. For the case where $\varepsilon=0, \delta=0.89$, the ignition times are $t_{\mathrm{ig}}=14.00$ and $t_{\mathrm{ig}}=12.04$ for the dynamic and static boundary conditions, respectively. Similarly, for $\varepsilon=0.027, \delta=0.92$ and the static boundary condition $t_{\mathrm{ig}}=12.40$, whilst with the dynamic boundary condition $t_{\mathrm{ig}}=11.67$. This indicates that, for a given stockpile, the ignition time is different once the dynamic boundary condition is applied.

In two dimensions, Table 3 shows that the effects of the dynamic boundary condition are more prominent than in the one-dimensional case, with a larger reduction in the critical FK parameter observed when the oscillations are added. For the $\varepsilon=0$ case, the oscillations cause stockpiles to ignite that are subcritical when considering a static boundary condition. The one-dimensional stockpiles have a lower critical value so one would expect, as we increase the length $L_{x}$ of the two-dimensional stockpile with fixed height $L_{y}$, the critical parameter will decrease. This relationship is displayed in Figure 1 for both dynamic and static boundary conditions. 
Table 3: The critical FK parameter $\delta_{\text {cr }}$ for a two-dimensional rectangular domain with $u_{0}=0.637, \omega=0.3$ and $\phi=0$.

\begin{tabular}{lcccc}
$\varepsilon$ & Oscillations & $t_{f}=0.3$ & $t_{f}=1$ & $t_{f}=10$ \\
\hline 0 & no & 4.072 & 2.20 & 1.71 \\
0 & yes & 3.615 & 2.14 & 1.69 \\
0.027 & no & 4.294 & 2.3 & 1.76 \\
0.027 & yes & 3.843 & 2.23 & 1.75
\end{tabular}

Figure 1: The critical FK variable $\delta_{\text {cr }}$ for ignition to occur within a year, as the ratio of side length to height is increased with $\epsilon=0.027$ and $\phi=0$.

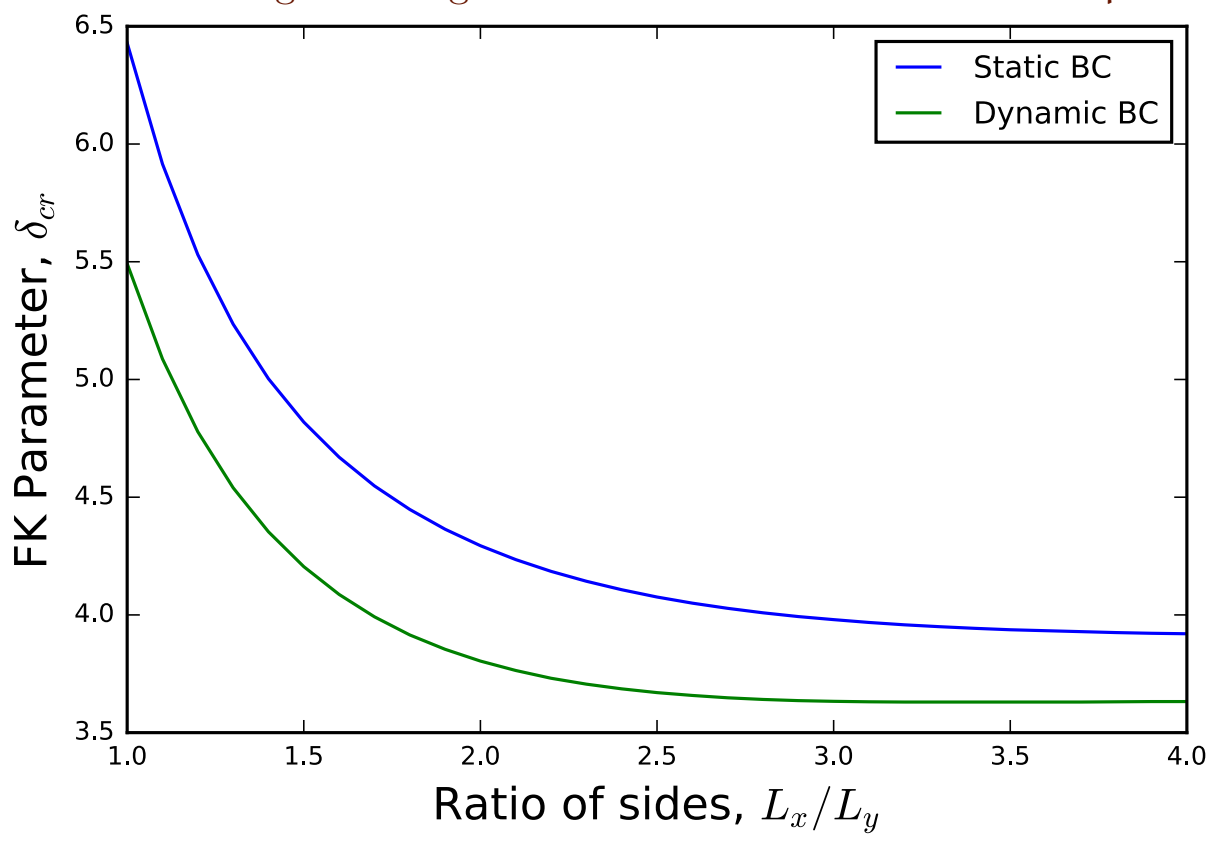


Figure 2: The effects of changing the stockpile construction time $\phi$ on the critical FK parameter $\delta_{\mathrm{cr}}$, for stockpile ignition within a year $\left(t_{\mathrm{f}}=0.3\right)$.

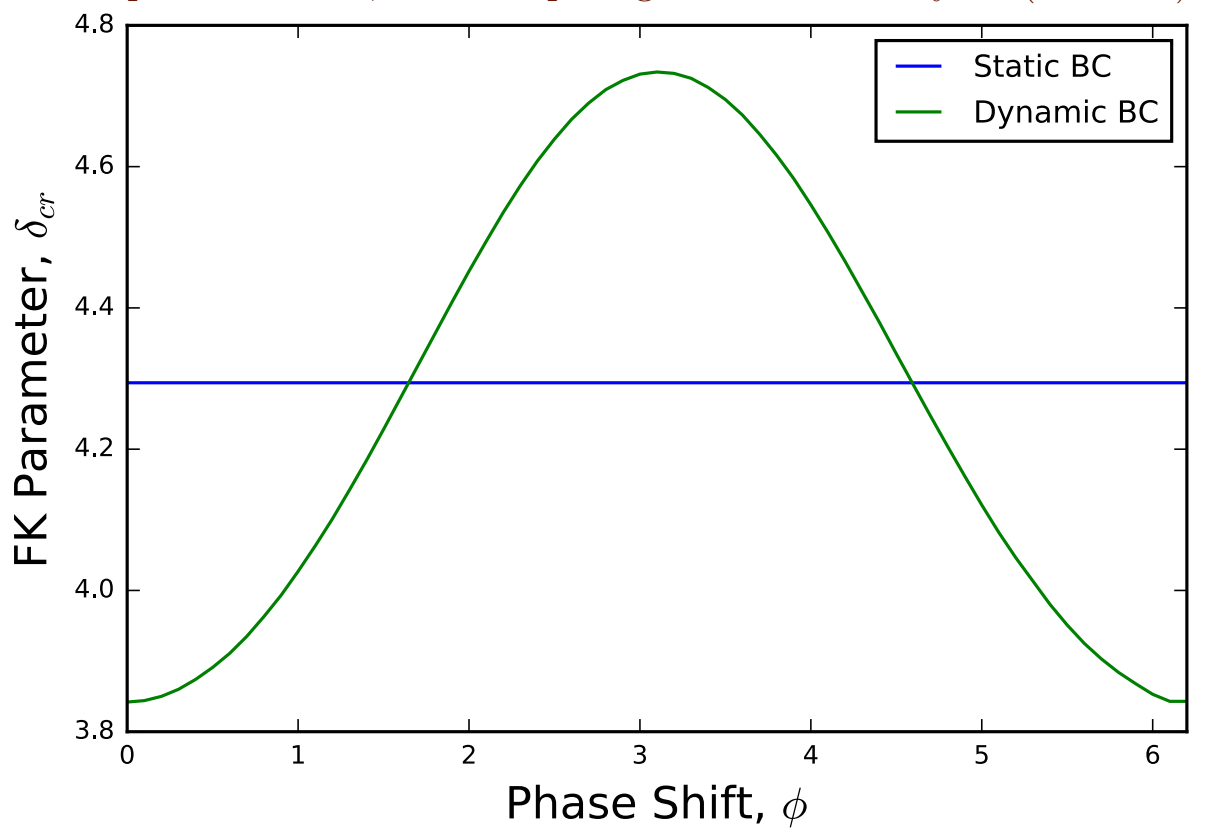

So far our analysis has assumed the stockpiles are constructed at a specific time of year, specifically spring where $\phi=0$. For $t_{f}=0.3$, Figure 2 indicates which stockpiles will ignite within a year. Certain stockpiles constructed in spring will ignite whilst identical ones constructed in autumn will not.

When the final time is increased to $t_{f}=1$, Figure 3 shows that the critical FK parameter becomes less sensitive to the construction date. Another key observation from comparing Figures 2 and 3 is that on increasing $t_{f}$ there are more starting points where the critical parameter is lower than the static boundary condition. Figure 3 also shows a shift in the oscillation peak towards $\phi=0$.

We now consider the ignition times as the FK parameter $\delta$ is varied. Figure 4 
Figure 3: The effects of changing the initial construction time $\phi$ on the critical FK parameter $\delta$ for a final time $t_{f}=1$, corresponding to just over a three year period.

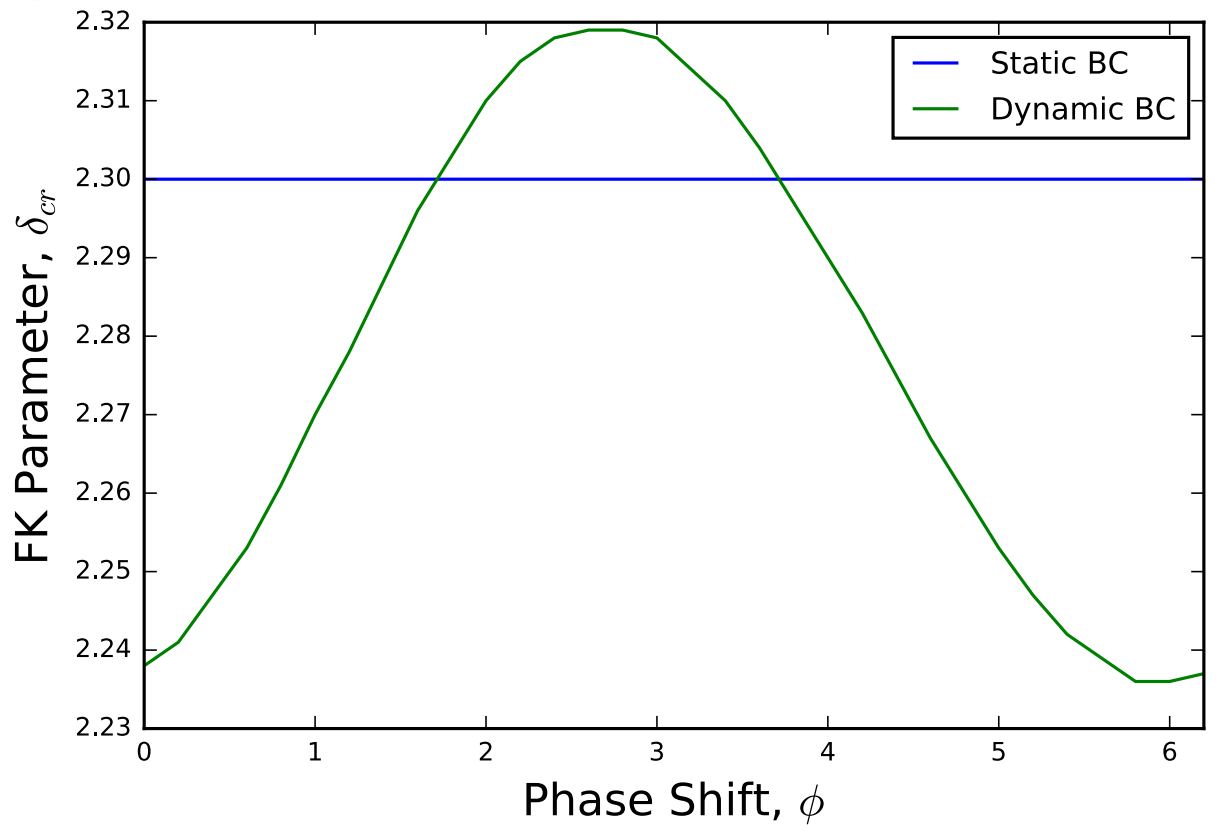

compares the ignition times for the static boundary condition with the dynamic boundary condition. Stockpiles constructed in spring, $\phi=0$, are plotted along with stockpiles constructed half a year later, $\phi=\pi$. For the larger values of the FK parameter, there is a distinct difference between the ignition times in each case. For smaller values of the FK parameter the ignition times for stockpiles constructed later $(\phi=\pi)$ are lower than those with the static boundary condition. This indicates that, as we consider longer periods of time, the dynamic boundary condition reduces the ignition times. Figure 4 suggests that there will exist some time such that, regardless of when the stockpile is built, the effect of the dynamic boundary condition will cause the stockpile to ignite earlier than is the case for the static boundary condition. 
Figure 4: The ignition times as the FK parameter $\delta$ is varied.

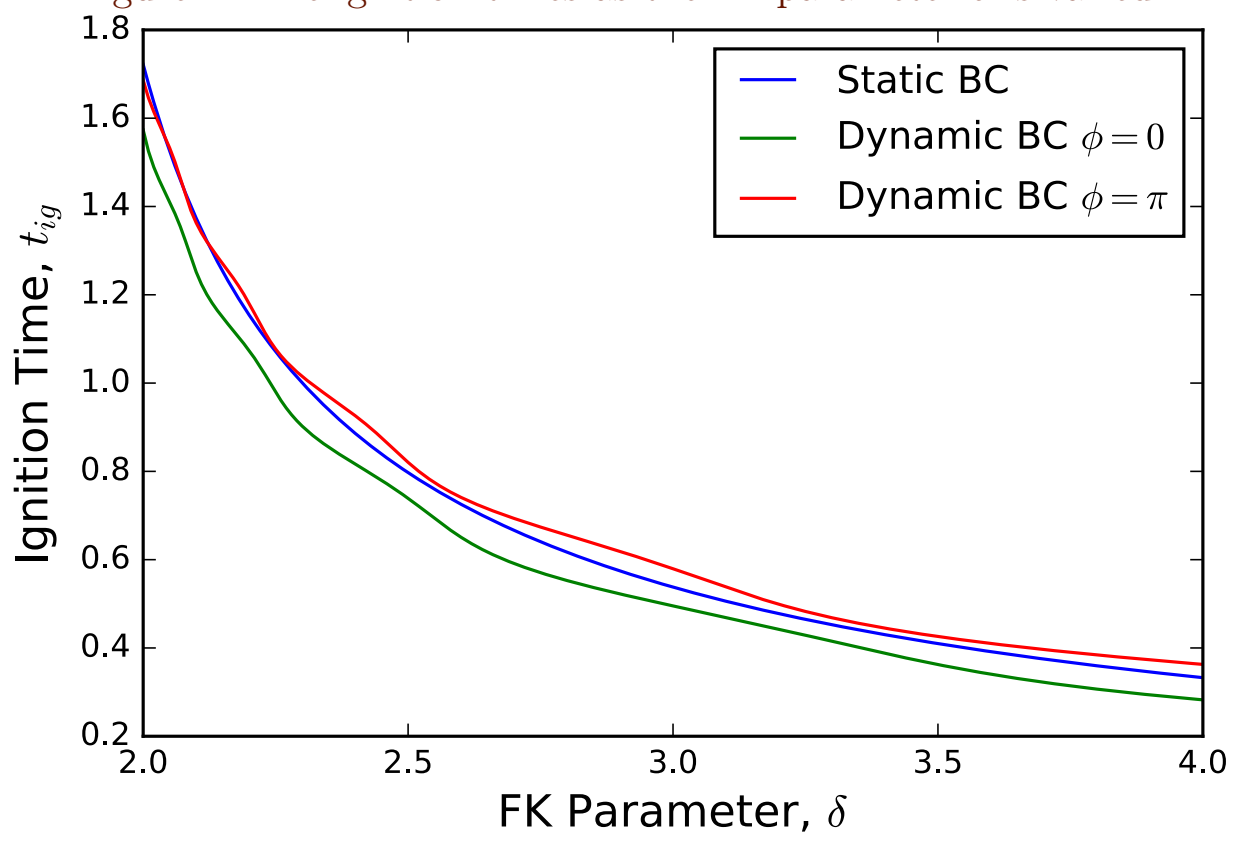

\section{Numerical validation}

The numerical scheme used was a finite difference scheme which is second order in space and first order in time, with an interval width of 0.02 and a time step of $5 \times 10^{-6}$. The values obtained for the ignition times are dependent on the type of numerical scheme used. It is important to show that our numerical scheme converges to the true solution. We validate the scheme by comparing numerical solutions with different interval widths. We compared the solutions with 50,100, 200, 400, 800 intervals for the one dimensional model, to the solution with 1600 intervals. By halving the interval width at each step, the solution with 1600 intervals has common nodes with the solutions corresponding to each other interval width. We use this to determine 
Figure 5: The $\log _{2}$ (Error) of the numerical scheme in the spatial variable at time $t=1$.

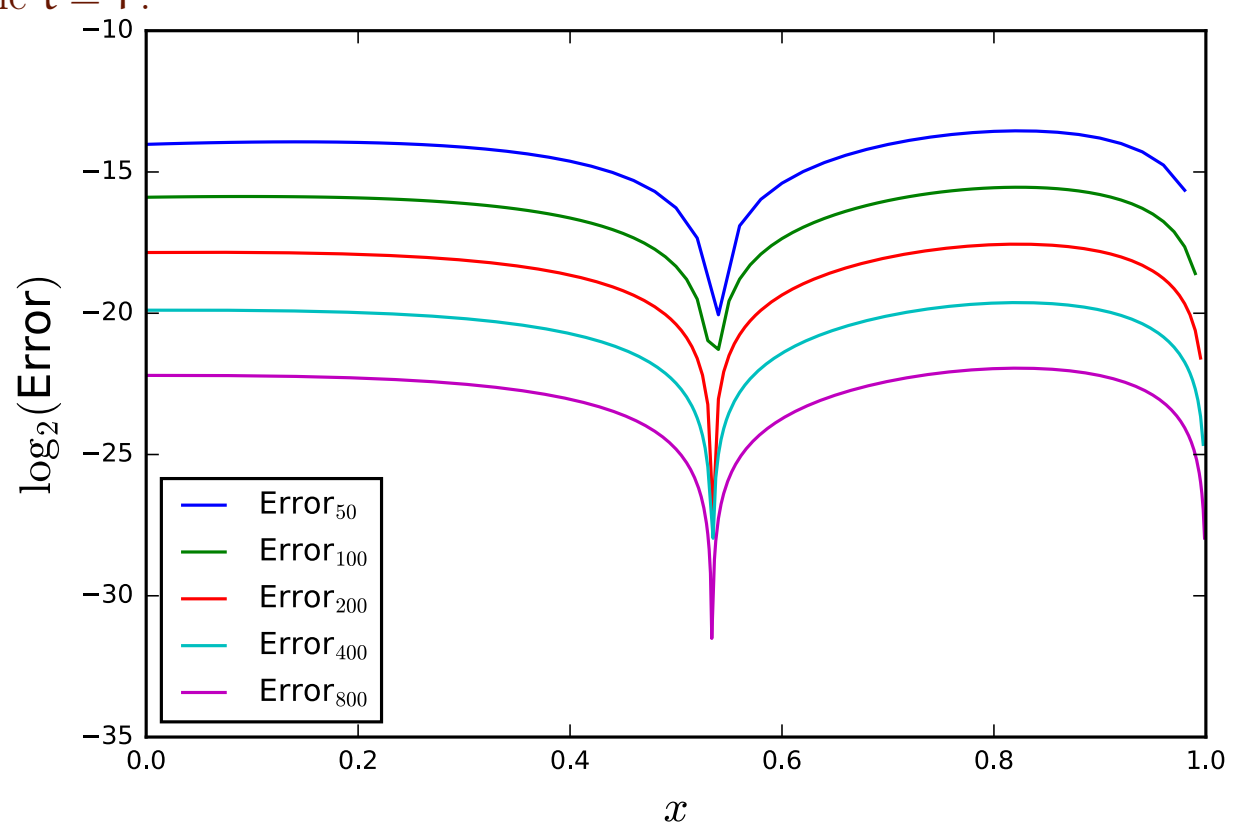

a pointwise convergence at each node. The resulting error vector is

$$
\text { Error }_{i}=\left|u_{1600, i}-u_{i}\right|
$$

where $\mathfrak{u}_{1600, i}$ is the solution vector with 1600 intervals restricted to the common nodes of the solution vector with $i=50,100,200,400,800$ intervals. Figure 5 plots each error and indicates that as we halve the interval width, the solutions converge to the solution provided by the most refined grid. A constant difference at each step indicates that the error is reduced by the same factor. This is expected as the interval width is being halved at each step. This suggests that the numerical scheme does not have any unexpected behaviour. We also check convergence in time. We selected a point at the centre of the domain and tracked the temperature over time. At each successive iteration 
Figure 6: The $\log _{2}$ (Error) of the finite difference scheme at a point in the centre of the domain.

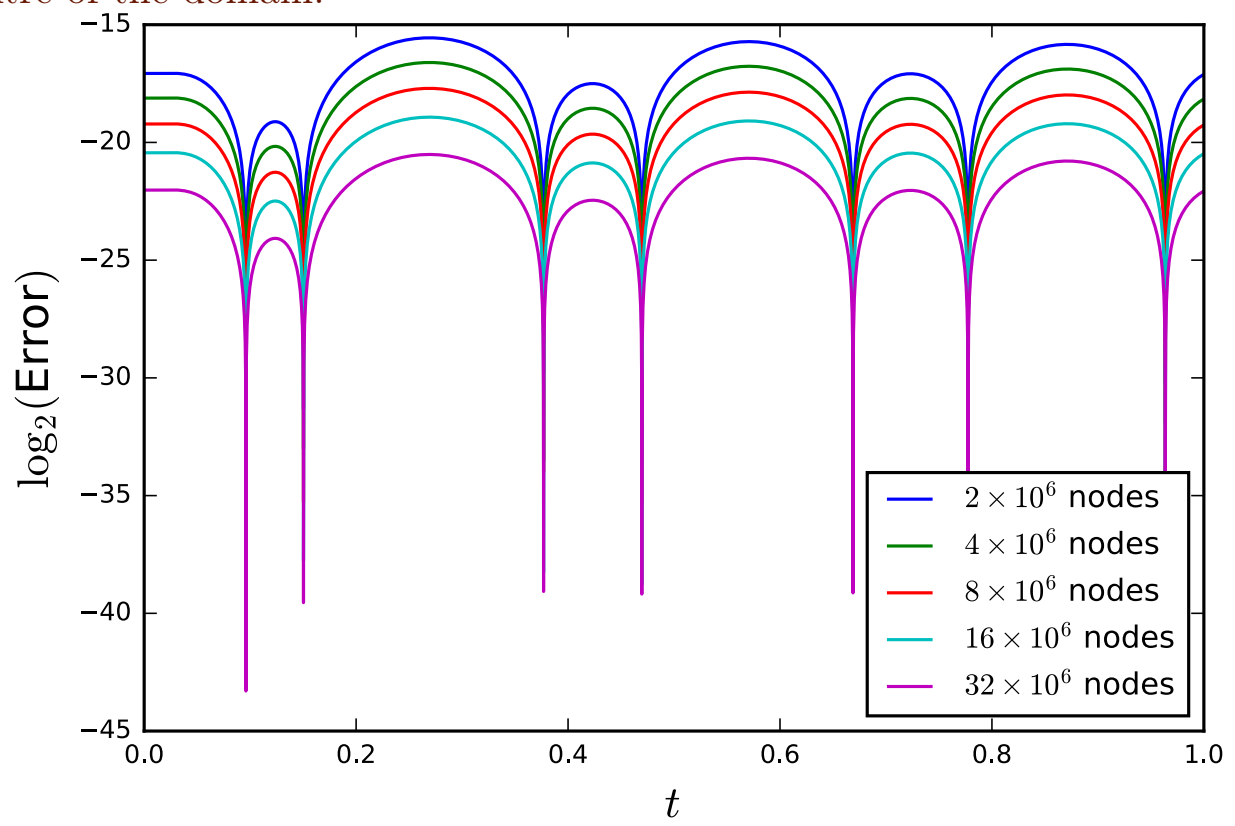

the time interval was halved and the temperature compared to simulation with the shortest time interval. Figure 6 displays how the solution converges in time.

Figures 5 and 6 indicate that the numerical schemes are converging in a consistent manner. This provides some validation for our numerical algorithm.

\section{Conclusion}

The dynamic boundary condition has a significant effect on the ignition times of stockpiles. Stockpiles that are considered subcritical with the static boundary condition, are supercritical with the dynamic boundary condition. 
Our numerical investigation demonstrates that identical stockpiles can ignite at different times, depending on the stage of the seasonal cycle they are constructed. Our study suggests that stockpiles built in spring will ignite quicker than those constructed at other times, whilst those constructed in autumn will take the longest time to ignite.

The effects considered in this study are limited to seasonal temperature variation. The effect of daily temperature variation was not considered. The long term simulations conducted suggest that, after extended periods of time, oscillations may reduce the critical values of the FK parameter, for all start times. This suggests that daily variations may reduce the critical parameters.

Acknowledgements This work was conducted with the assistance of an Australian Government Research Training Program award.

\section{References}

[1] P. C. Bowes. Self-heating; evaluating and controlling the hazards. Dept. of the Environment, Building Research Establishment, 1984 (cit. on pp. C46, C49, C50).

[2] R. J. Longbottom, B. J. Monaghan, G. Zhang, D. J. Pinson, and S. J. Chew. "Self-sintering of BOS Filter Cake for Improved Recyclability". In: ISIJ Int. 59.3 (2019), pp. 432-441. DOI: 10.2355/isijinternational. ISIJINT-2018-627 (cit. on p. C46).

[3] V. Novozhilov. "Thermal explosion in oscillating ambient conditions". In: Sci. Rep. 6 (July 2016), p. 29730. DOI: 10.1038/srep29730 (cit. on p. C47).

[4] N. C. Roy. "Convection characteristics in a closed vessel in the presence of exothermic combustion and ambient temperature oscillations". In: Int. J. Heat Mass Trans. 116 (2018), pp. 655-666. DOI:

10.1016/j.ijheatmasstransfer.2017.09.058 (cit. on p. C47). 


\section{Author addresses}

1. Matthew Berry, School of Mathematics and Applied Statistics, University of Wollongong, Wollongong, NSW 2522 AUSTRALIA orcid:0000-0001-7896-2478

2. Mark Nelson, School of Mathematics and Applied Statistics, University of Wollongong, Wollongong, NSW 2522 AUSTRALIA

3. Brian Monaghan, School of Mechanical, Materials, Mechatronic and Biomedical Engineering and ARC Research Hub for Australian Steel Manufacturing, University of Wollongong, Wollongong, NSW 2522 Australia

4. Ben Whale, School of Mathematics and Applied Statistics, University of Wollongong, Wollongong, NSW 2522 AUsTRALIA 